\title{
BMJ Open Effects of sulforaphane on cognitive function in patients with frontal brain damage: study protocol for a randomised controlled trial
}

\author{
Fangkun Liu, ${ }^{1}$ Jing Huang, ${ }^{2}$ Gangrui Hei, ${ }^{2}$ Renrong Wu (D) , ${ }^{2}$ Zhixiong Liu (D) ${ }^{1}$
}

To cite: Liu F, Huang J, Hei G, et al. Effects of sulforaphane on cognitive function in patients with frontal brain damage: study protocol for a randomised controlled trial. BMJ Open 2020;10:e037543. doi:10.1136/ bmjopen-2020-037543

- Prepublication history for this paper is available online. To view these files, please visit the journal online (http://dx.doi. org/10.1136/bmjopen-2020037543).

$\mathrm{FL}$ and $\mathrm{JH}$ contributed equally and share first co-authorship.

Received 07 February 2020 Revised 04 August 2020 Accepted 23 August 2020

Check for updates

(C) Author(s) (or their employer(s)) 2020. Re-use permitted under CC BY-NC. No commercial re-use. See rights and permissions. Published by BMJ.

For numbered affiliations see end of article.

Correspondence to Dr Zhixiong Liu; zhixiongliu@csu.edu.cn and Dr Renrong Wu; wurenrong@csu.edu.cn

\section{ABSTRACT}

Introduction Many patients with frontal brain damage show serious cognitive function deficits, which hamper their quality of life and result in poor clinical outcomes. Preclinical research has shown that sulforaphane can significantly improve spatial localisation and working memory impairment after brain injury. The primary aim of this double-blind randomised controlled clinical trial is to assess the efficacy of sulforaphane for improving cognitive function in patients with frontal brain damage.

Methods and analysis Ninety eligible patients will be randomly allocated to an active treatment or a placebo group in a 2:1 ratio. Participants will undergo a series of cognitive and neuropsychiatric tests at baseline (week 0 ) and after 12 weeks to determine the effect of sulforaphane on cognition. Magnetic resonance spectrum of the brain will be studied using the $3 \mathrm{~T}$ MRIs of the brain to detect brain metabolites markers, including $\mathrm{N}$-acetyl aspartate, glutamate (Glu), glutathione (GSH) and $\gamma$-aminobutyric acid (GABA). Blood brain-derived neurotrophic factor, Glu, GSH and GABA levels and gut microbiota will also be assessed over this period. This study will also evaluate long-term outcomes of brain trauma, brain tumours and cerebrovascular disease via exploratory analyses. The primary outcome will be the difference in scores of a battery of cognitive tests after 12 weeks of sulforaphane treatment. The secondary outcomes will be changes in the Functional Activities Questionnaire (FAQ), the Patient Health Questionnaire (PHQ-9), the Self-Rating Anxiety Scale, the changes in T1-weighted MRI and resting-state functional MRI findings, and changes in brain and blood metabolic markers and gut microbiota at weeks 0 and 12. We expect that sulforaphane will yield favourable results in treating memory and learning deficits for patients with frontal brain damage. Cognitive functional treatment may also improve brain trauma, brain tumours and cerebrovascular outcomes.

Ethics and dissemination The study protocol has been approved by the Medical Ethics committee of the Xiangya Hospital of Central South University (No. 2017121019). The results will be disseminated in peer-reviewed journals and at international conferences.

Trial registration number This trial was registered on Clinicaltrials.gov on 31 January 2020 (NCT04252261). The protocol version is V.1.0 (20 December 2019).

\section{Strengths and limitations of this study}

- This trial will be the first randomised controlled trial to assess the effect of sulforaphane on the cognitive performance of patients with brain damage.

- The use of resting-state functional MRI and brain metabolite markers in conjunction with serum markers is unique and provides an innovative approach.

- The results of this study will help elucidate whether sulforaphane can be used in cognitive rehabilitation strategies in brain trauma, brain tumours and other types of brain damage.

- The various causes of frontal brain damage may affect the results of cognitive performance.

- This preliminary study will be limited to Chinese population, and studies on other population groups will subsequently be required.

\section{BACKGROUND}

The frontal brain, located in the front of the cerebral cortex, is involved in cognitive functions such as attention, working memory, language, spatial orientation and execution. ${ }^{1-3}$ Frontal lobe lesions resulting from conditions, such as trauma, tumour, cerebrovascular disease or parasitic disease, can damage the cortical networks and lead to local neuronal necrosis, degeneration, ischaemia, and inflammation, and cognitive deficits. ${ }^{14-6}$ Studies have shown that $42 \%-80 \%$ of the patients with frontal brain damage show serious cognitive function deficits, particularly in attention, learning, memory and executive function, and the damage can be sustained for more than 4-9 months. ${ }^{2}{ }^{7-10}$ In addition, the cognitive and functional impairment has been proved to be associated with poor clinical outcomes in patients with glioma and stroke. ${ }^{1112}$ Cognitive rehabilitation and treatment interventions can improve attention, executive function and decision-making abilities, but are poorly effective for abstract thinking, memory and visual structural 
abilities. ${ }^{913}$ As such, it is of great clinical significance to develop and study treatments for cognitive impairment after frontal lobe damage.

Sulforaphane, an active extract of cruciferous vegetables such as broccoli and cabbage, upregulates genes that suppress oxidative stress, inflammation and DNA damage. Sulforaphane also enhances mitochondrial function, promotes glutathione (GSH) synthesis and crosses the blood-brain barrier to reduce nerve inflammation. ${ }^{14}$ Sulforaphane has shown significant antioxidant and cellular protective effects in animal models associated with oxidative stress, such as focal cerebral ischaemia, brain inflammation and intracranial haemorrhage, ${ }^{16-19}$ and it could improve attention-focusing function in patients with schizophrenia. ${ }^{20}$ In rat models, sulforaphane can significantly improve spatial localisation and working memory impairment after traumatic brain damage by inhibiting oxidative stress. ${ }^{21}$ We, therefore, hypothesise that sulforaphane is useful as an early intervention to reduce neuroinflammation and improve cognitive function in patients with cognitive impairment after frontal lobe damage.

Previous studies have shown that neuron-specific metabolite abnormalities in N-acetyl aspartate (NAA), brain-derived neurotrophic factor (BDNF), GSH, glutamate (Glu) and $\gamma$-aminobutyric acid (GABA) levels could modulate and predict different aspects of cognitive performance. ${ }^{22-28}$ Research has shown that oxidative stress can act on BDNF in the injured brain to affect synaptic plasticity and cognition; ${ }^{29}$ therefore, the sulforaphane-mediated antioxidative effect might influence BDNF levels to improve cognitive function. Several studies also show that sulforaphane augments whole brain and peripheral GSH levels to attenuate oxidative stress, which also supports its potential application in cognitive improvement. ${ }^{30}$ Reportedly, gut microbiota is closely linked to memory, mood and cognitive function, through the interkingdom signalling system and the bidirectional communication of neuroendocrine and enteric nervous systems. ${ }^{32-34}$ Members of genera such as Escherichia, Lactobacillus and Trichuris release neurotransmitters and neuropeptides (including GABA and BDNF) to affect brain function by modulating signalling within the enteric nervous system. ${ }^{35}$ Probiotic administration has been shown to restore cognitive function and frontoparietal connectivity in various animal disease models, including hepatic encephalopathy, vascular dementia and cerebral ischaemia/reperfusion injury. ${ }^{36}$ On the basis of these theories, we will also assess brain and blood BDNF, NAA, GSH, Glu and GABA levels and gut microbiota over the study period to reveal possible mechanisms by which sulforaphane alters cognition performance.

\section{STUDY GOALS AND OBJECTIVES}

The primary hypothesis is that sulforaphane treatment improves cognitive function, especially learning and memory in patients with frontal brain damage. We will explore associations between changes in cognitive function and changes in functional MRI (fMRI) and T1-weighted MRI as well as changes in the brain and blood BDNF, NAA, Glu, GSH and GABA levels. The associations between changes in cognitive function and gut microbiota over time will also be investigated. Finally, we will assess the effect of the improvement of in cognitive function on brain trauma, brain tumours and cerebrovascular outcomes in an exploratory analysis.

\section{METHODS AND ANALYSIS}

\section{Patient and public involvement}

Patients and public were not involved in the development of the research questions, study design or study conduct.

\section{Study design}

This is a prospective, single-centre, randomised controlled clinical trial that will be held at the Xiangya Hospital, Central South University, Changsha, China. Study recruitment will begin on 31 May 2020 and the estimated study completion date for the primary outcomes is 31 May 2022. We aim to assess 90 participants using a series of cognitive tests at baseline and 12 weeks later to determine the effect of sulforaphane (compared with placebo) on the cognition of patients with frontal brain damage. Baseline would be at least 2 days after surgery and less than 21 days after surgery. Participants will also be imaged using 3T MRI at these time points to obtain T1-weighted MRI, and resting-state fMRI (rs-fMRI), and to determine the MRI markers of brain metabolites, including NAA, Glu, GSH and GABA. Blood BDNF, Glu, GSH and GABA levels will also be assessed over this period. Gut microbiome and microbial DNA will be extracted from a gut sample according to the standard protocol. ${ }^{37}$ After that, bacterial 16S rRNA gene sequencing and metagenomic technique will be applied to assess the changes in gut microflora over time. Figure 1 depicts the design of the study.

\section{Subjects and recruitment}

We will recruit 90 patients aged 18-65 years, and of both sexes, who have incurred cognitive deficits after frontal brain damage. All participants will be recruited from the Neurosurgery Department at the Xiangya Hospital between 10 July 2020 and 31 May 2022. Frontal brain injury will be verified using CT or MRI. Cognitive deficits will be diagnosed using the Chinese version of the Montreal Cognitive Assessment (MoCA-C), which is an eligibility test to assess multiple cognitive domains. ${ }^{38} 39$ Participants scoring $<26$ will be assessed as having cognitive deficits $(<25$ for patients who have received education for $<12$ years) ${ }^{39}$ All eligible recruited patients will then be randomly allocated to either the sulforaphane group or the placebo group in a 2:1 ratio. During the trial period, standard clinical treatment and follow-up will be used to measure patient conditions and maintain compliance. All patients must be able to answer a series of cognitive tests, and complete measures including cognitive tests, the 


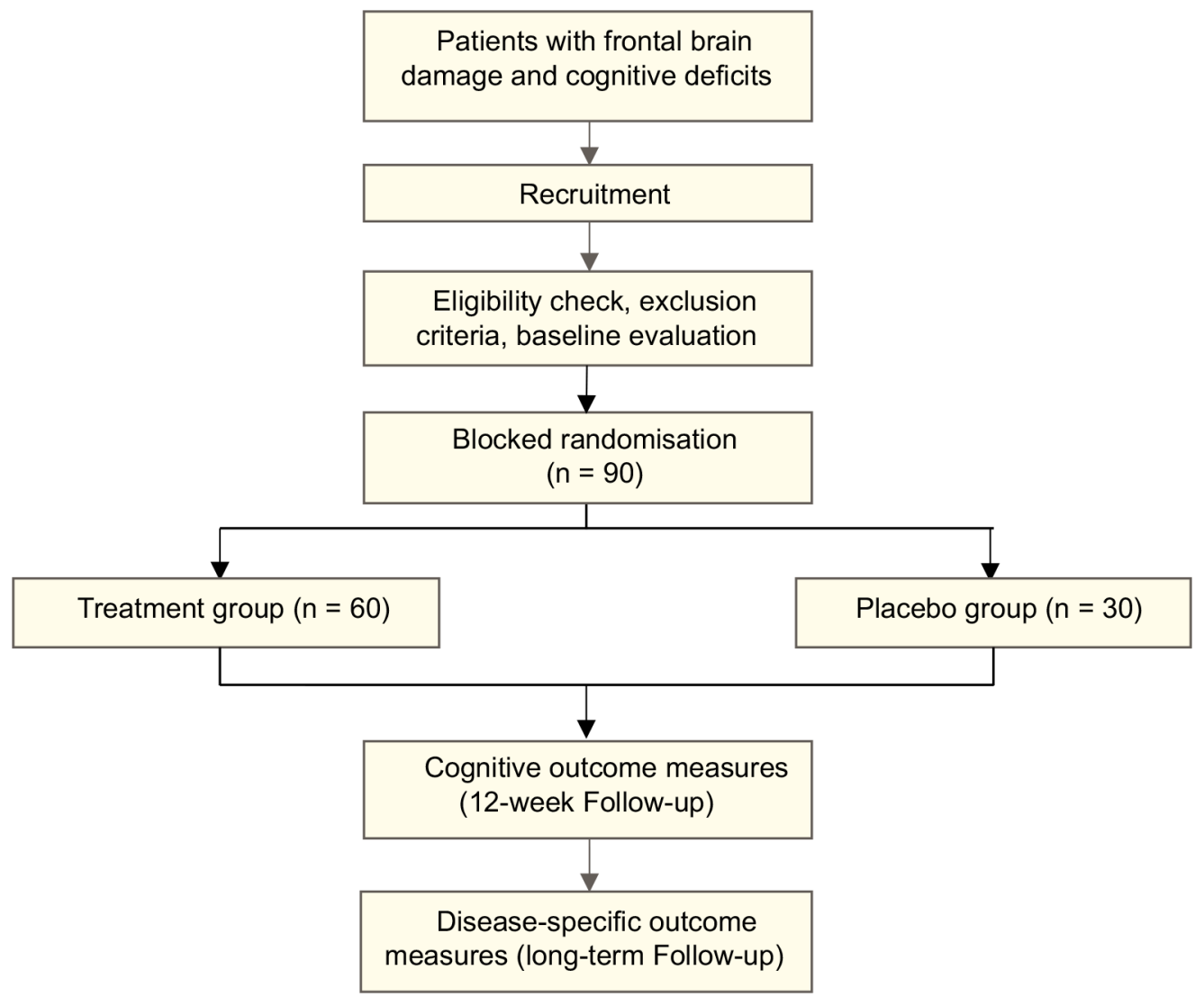

Figure 1 Study flow diagram: after recruitment, eligibility check and baseline evaluation, 90 participants will be randomised to sulforaphane group or placebo group in a 2:1 ratio. Baseline and 12-week follow-up evaluation include neurocognitive evaluation, T1-weighted MRI, resting-state functional MRI, magnetic resonance spectrum, Blood brain-derived neurotrophic factor, $\mathrm{N}$-acetyl aspartate, glutathione, glutamate and $\gamma$-aminobutyric acid levels and gut microbiota. Long-term outcomes for brain trauma, brain tumours and cerebrovascular disease will also be assessed.

Functional Activities Questionnaire (FAQ), the Patient Health Questionnaire-9 (PHQ-9), the Self-Rating Anxiety Scale (SAS), MRI of the brain, blood metabolites and gut microbiota. After 12-week double-blind treatment phase, participants will be encouraged to follow-up until 24 weeks for further cognitive evaluation.

\section{Exclusion criteria}

1. History of cognitive impairment by clinical diagnosis, such as dementia and intellectual disability.

2. History of seizures owing to the potential of sulforaphane to increase seizure susceptibility. ${ }^{40}$

3. A current or prior significant DSM-5 (Diagnostic and Statistical Manual of Mental Disorder, Fifth Edition) diagnosis of psychiatric disease, chronic neurological disorders or active substance abuse.

4. Inability to cooperate while undergoing MoCA-C Test owing to disturbances in consciousness or mental disorder.

5. Pregnancy or lactation.

6. Life expectancy $<3$ months.

7. CO (carbon monoxide) poisoning, autoimmune encephalitis, intracranial infection or other types of diffuse encephalopathies.

8. Plan to receive radiotherapy during the trial period.
9. Laboratory examination showing liver and kidney insufficiency or other severe complications; presence of diseases which may interfere with the results of the evaluation.

10. Contraindications to MRI scanning, such as metallic implants or prosthetics, pacemakers, dependence on benzodiazepine medication, prohibitive claustrophobia or other medical conditions with potential safety risk.

11. Involvement in other trials 1 month prior to the start of the trial or during the trial period

\section{Randomisation}

Block randomisation will be conducted using the SAS (vesion is v9.4 software. Ninety patients will be randomised in a 2:1 ratio into a treatment group and a placebo group. Neuropsychologists, data analysts and patients will be blinded to the group assignment in this trial. Only two of the authors involved with the block randomisation will assign participants to interventions.

\section{Study intervention}

Sulforaphane or an identical placebo medication will be used as a treatment intervention. Patients will be given a specific number of sulforaphane tablets (Avmacol, 60 
Table 1 Overview of cognitive tests, MRls of the brain, magnetic resonance spectrum, blood metabolites and gut microbiota tested in the study

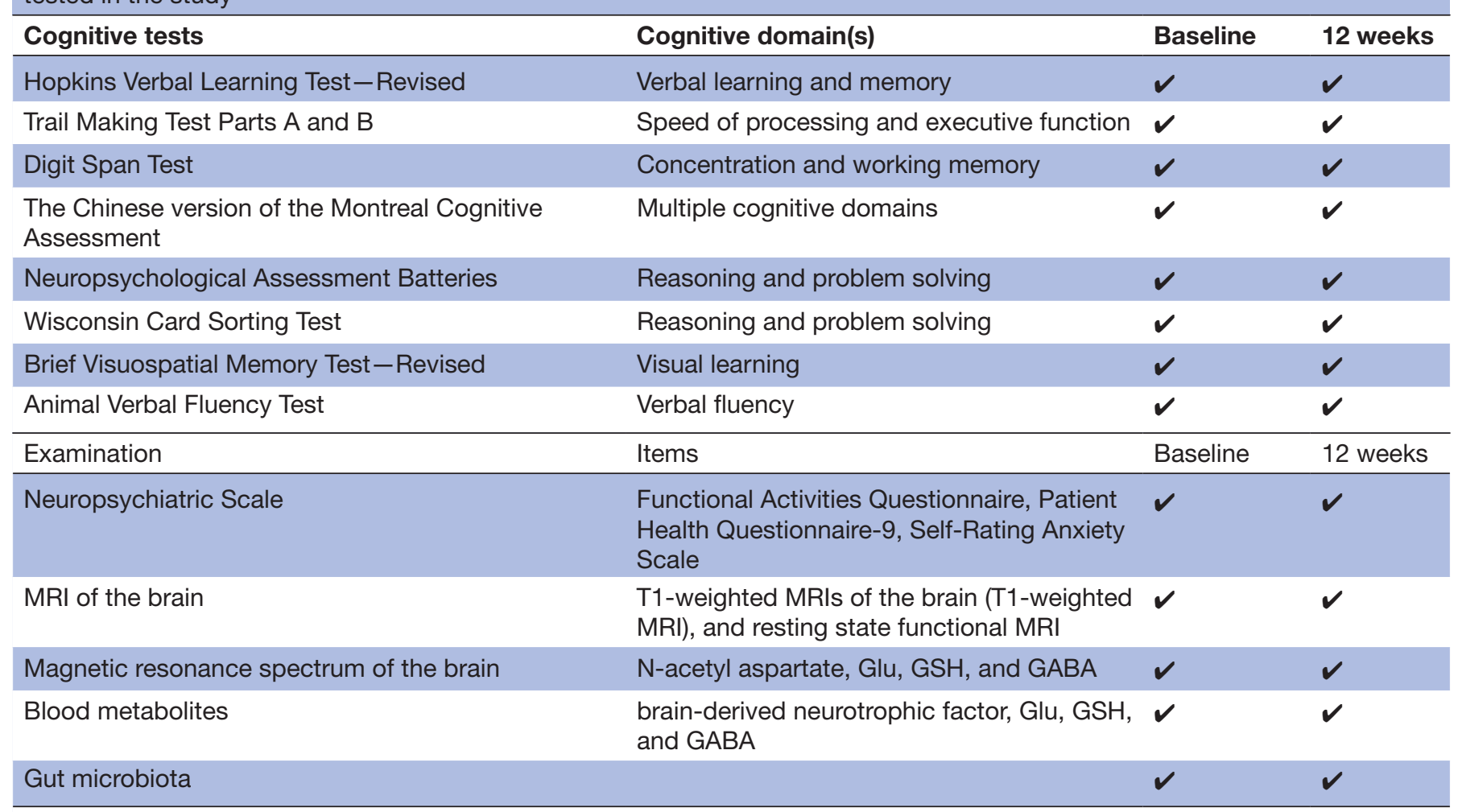

GABA, $\gamma$-aminobutyric acid; Glu, glutamate; GSH, glutathione.

tablets) once a day with dosage adjusted according to body weight, with patients weighting $<45 \mathrm{~kg}$ will receive two tablets $(1750 \mathrm{mg}$ ) per day, patients weighing $45-90 \mathrm{~kg}$ will receive three tablets $(2550 \mathrm{mg})$ per day and patients weighing $>90 \mathrm{~kg}$ will receive four tablets $(3400 \mathrm{mg}$ ) per day. Study medication will be dispensed at baseline. For standard care, all patients will attend their clinical follow-up at the Xiangya Neurosurgery Outpatient Center independent of the study and group assignment and will be examined by other physicians not involved in this study.

\section{Measures}

Outcome measures for the study, including cognitive and neuropsychiatric tests, MRI of the brain and magnetic resonance spectrum (MRS) of the brain findings, blood metabolite levels and gut microbiota, are listed in table 1.

\section{Cognitive testing}

Cognitive function tests will be performed at baseline and again at 12 weeks after randomisation. These include the following:

1. Hopkins Verbal Learning Test-Revised to assess verbal learning and memory. ${ }^{41}$

2. Trail Making Test Parts A and B to assess the speed of processing and executive function. ${ }^{42}$

3. Digit Span Test to measure concentration and working memory. ${ }^{43}$

4. The MoCA-C to assess multiple cognitive domains. ${ }^{38} 39$
5. Neuropsychological Assessment Batteries to assess reasoning and problem solving. ${ }^{44}$

6. Wisconsin Card Sorting Test to detect frontal lobe dysfunction, and assess reasoning and problem solving. ${ }^{45}$

7. Brief Visuospatial Memory Test-Revised to measure visual learning. ${ }^{46}$

8. Animal Verbal Fluency Test to assess verbal fluency and frontal brain function. ${ }^{47}$

\section{MRIs of the brain}

All MRIs will be performed using a 3T Skyra scanner (Siemens, Erlangen, Germany). The MRI examination takes approximately $30 \mathrm{~min}$ to complete and comprises standardised sequences used for the analysis of brain morphometry, microstructure and function, including:

1. T1-weighted MRI of the brain, grey and white matter morphometry and anatomical reference.

2. rs-fMRI to investigate the functional connectivity between the prefrontal cortex (PFC) and other brain regions.

3. MRS with spectra recorded from the PFC;

Processing and interpretation of neuroimaging data will be conducted by an author who will be blinded to participant information and intervention. All scans will be reviewed and reported by a neuroradiologist.

\section{Sample size}

Sample size calculation will be carried out using the SAS software. Since there were no previous studies of 
sulforaphane in patients with frontal brain damage, we estimated the sample size based on our unpublished study of sulforaphane treatment on cognitive impairment for other diseases (ClinicalTrials.gov: NCT02880462). 90 was calculated as the number necessary to detect an average difference of 18.5 in multiple cognitive domains between baseline and 3 months in the treatment group, and of 11.9 in the placebo groups at $80 \%$ power with a twosided $\mathrm{p}$ value of 0.05 . These calculations include a $20 \%$ inflation for dropout and loss of patients to follow-up. $\mathrm{SD}$ in total scores are estimated at 14 at 0 and 3 months. However, without knowledge of the within-person correlation between data points, it is impossible to predict the increase in power calculation.

\section{Trial status}

Trial status is currently in the preparation phase for recruitment as of 16 May 2020.

\section{Safety considerations}

There are negligible risks for patients treated with sulforaphane (Avmacol, 60 tablets), which meets the requirements of the US Food and Drug Administration. Serious adverse events will be recorded and properly managed, and then reported to the Ethics committee of the Xiangya Hospital.

\section{Follow-up}

Outcome measures including cognitive and neuropsychiatric tests, T1-weighted MRI, rs-fMRI, brain and blood metabolic markers and gut microbiota, will be tested after the 12-week double-blind treatment phase. This study will also evaluate long-term outcomes for brain trauma, brain tumours and cerebrovascular disease via exploratory analyses. All patients in the double-blind treatment phase will be encouraged to be re-evaluated at week 24 . Study medicine will not be administered from week 12 to week 24. Further clinical treatment will be arranged by the study investigator and/or the subject's treating physician during this period. Participants who discontinue the intervention will be followed up for further statistical analysis according to the protocol because they have been randomly allocated.

\section{Data management and statistical analysis}

Every participant will receive a unique identification number for the duration of the study to maintain treatment anonymity. Neuropsychologists, data analysts and patients will be blinded to the treatment assignment in this trial. Only two of the authors involved with the block randomisation will assign participants to interventions. All cognitive, MRI, blood metabolite and sequencing data will be collected and stored by direct members of the research team before the end of the trial.

Statistical analysis will be performed from the obtained results by using SPSS V.24. The results of the continuous scale will initially be evaluated for normality and logarithmic conversions will be performed where appropriate. Results changes over time will be determined by mixed linear or non-linear longitudinal modelling, to determine whether each group behaves differently over time. Additional sensitivity analyses will be performed based on known predictors of the outcome (age, sex and other risk factors) and any baseline imbalances between groups. Subgroup analysis was conducted according to the causes of frontal brain injury. A two-sided $p$ value of 0.05 or less will be considered to be statistically significant. The relationship between changes in brain and blood GSH, GABA, NAA and BDNF levels, gut microbiota, and cognitive function will also be determined using the mixed linear longitudinal model for cognitive function measurements over time.

\section{Quality assurance}

Test assessors for these protocols undergo standard training and assessment and are recertified annually. Annual monitoring will also be conducted by a senior neuropsychologist. This trial will adhere to Consolidated Standards of Reporting Trials guideline.

\section{Outcomes and measurements \\ Primary outcome}

The difference in scores of a battery of cognitive tests after sulforaphane treatment between week 12 and week 0 .

\section{Secondary outcomes}

Besides evaluating the efficacy of sulforaphane on the cognitive performance of patients with frontal brain damage, we will analyse the changes in the FAQ the PHQ-9, the SAS, the changes in T1-weighted MRI findings, rs-fMRI findings and changes in gut microbiota and metabolic markers (including BDNF, GSH, GABA, Glu and NAA) in the central and peripheral expressions at week 0 and week 12 . We will determine whether any brain and blood metabolite changes are associated with changes in cognitive function and whether cognitive function is associated with altered brain structure and basic function.

\section{Duration of the project}

Patient recruitment was started on 31 May 2020 and will be completed by approximately 30 May 2022 .

\section{Ethics and dissemination}

The study protocol has been approved by the Medical Ethics committee of the Xiangya Hospital of Central South University (No. 2017121019). All substantial amendments to the protocol should be reviewed by the medical ethics committee.

Written informed consent will be obtained from the patient's legal representative and/or the patients. Before inclusion, the investigator or an authorised member of the study will explain to the potential subjects and their legal representatives about the purpose of the study, the methods, strict data storage protocol, reasonably anticipated benefits and the potential hazards of the study. Subjects will be informed about the study's voluntary nature that they have the right to cease participation at any time. Choosing not to participate will not in any way 
prejudice the care that the subject will receive for the treatment of his or her disease. The investigator will give sufficient time for the subject to read the consent form, ask questions and make decisions. Patients will only be included if their legal representatives provide written informed consent.

The results of this study will be disseminated via an international peer-reviewed publication. Further, it will be presented at national and international conferences as posters or oral presentations for communication and discussion.

\section{DISCUSSION}

This trial, to the best of our knowledge, is the first randomised controlled trial to assess the effect of sulforaphane on the cognitive performance of patients with brain damage. This is important as cognitive performance impairment leads to poor quality of life and has previously been shown to increase the risk of clinically significant outcomes and predict poor cancer-related outcomes. This study will also ascertain the clinical significance of sulforaphane by measuring the improvement in cognitive outcomes and by quantifying the relationship between cognitive outcomes and changes in brain fMRI and T1-weighted MRI, as well as with changes in brain and blood BDNF, NAA, Glu, GSH and GABA levels, and gut microbiota. In addition, we will assess the effect of cognitive function improvement in brain trauma, brain tumours and cerebrovascular outcomes in an exploratory analysis.

\section{Author affiliations \\ ${ }^{1}$ Department of Neurosurgery, Central South University (CSU), 410008, Changsha, Hunan, China \\ ${ }^{2}$ Department of Psychiatry, the Second Xiangya Hospital, Central South University, 410011, Changsha, Hunan, China; Mental Health Institute of the Second Xiangya Hospital, Central South University, Chinese National Clinical Research Center on Mental Disorders (Xiangya), Chinese National Technology Institute on Mental Disorders, Hunan Key Laboratory of Psychiatry and Mental Health, 410011, Changsha, Hunan, China}

Acknowledgements The investigators would like to acknowledge the valued contribution of the participants and the support from their general practitioners.

Contributors FL provided input into design for statistical analysis of cognitive data. $\mathrm{JH}$ and RW were involved in study design, protocol preparation and acquisition of funding. MRI of the brain, brain and blood metabolites assessment, gut microbial DNA extraction, gene sequencing and cognitive assessments were carried out by experienced research assistants and test assessors. GH generated the random allocation sequence and assigns participants to interventions. FL was responsible for the trial protocol draft and final revision and JH had prepared MRI protocol details. RW and ZL were responsible for the project concept. All authors have reviewed and provided critical revision of the manuscript.

Funding This work was supported by the National Natural Science Foundation of China under grant no. 81873635 and 81901401, and the Natural Science Foundation for Young Scientist of Hunan Province, China (Grant No. 2019JJ50952).

Competing interests None declared.

Patient consent for publication Not required.

Provenance and peer review Not commissioned; externally peer reviewed.

Open access This is an open access article distributed in accordance with the Creative Commons Attribution Non Commercial (CC BY-NC 4.0) license, which permits others to distribute, remix, adapt, build upon this work non-commercially, and license their derivative works on different terms, provided the original work is properly cited, appropriate credit is given, any changes made indicated, and the use is non-commercial. See: http://creativecommons.org/licenses/by-nc/4.0/.

\section{ORCID iDs}

Renrong Wu http://orcid.org/0000-0002-5804-6648

Zhixiong Liu http://orcid.org/0000-0003-3733-7259

\section{REFERENCES}

1 Stuss DT, Benson DF. Neuropsychological studies of the frontal lobes. Psychol Bull 1984;95:3-28.

2 Stuss DT, Gallup GG, Alexander MP. The frontal lobes are necessary for 'theory of mind'. Brain 2001;124:279-86.

3 Gläscher J, Tranel D, Paul LK, et al. Lesion mapping of cognitive abilities linked to intelligence. Neuron 2009;61:681-91.

4 Walker KR, Tesco G, GJFian T. Molecular mechanisms of cognitive dysfunction following traumatic brain injury. Front Aging Neurosci 2013;5:29.

5 Abdul-Muneer PM, Chandra N, Haorah J. Interactions of oxidative stress and neurovascular inflammation in the pathogenesis of traumatic brain injury. Mol Neurobiol 2015;51:966-79.

6 Cheng $\mathrm{Q}$, Jiang $\mathrm{B}, \mathrm{Xi}$ J, et al. The relation between persistent coma and brain ischemia after severe brain injury. Int $J$ Neurosci 2013;123:832-6.

7 Tucha O, Smely C, Preier M, et al. Cognitive deficits before treatment among patients with brain tumors. Neurosurgery 2000;47:324-34. discussion 333-324.

8 Badre D. Cognitive control, hierarchy, and the rostro-caudal organization of the frontal lobes. Trends Cogn Sci 2008;12:193-200.

9 Tucha O, Smely C, Preier M, et al. Preoperative and postoperative cognitive functioning in patients with frontal meningiomas. $J$ Neurosurg 2003;98:21-31.

10 Wheeler MA, Stuss DT, Tulving E. Frontal lobe damage produces episodic memory impairment. J Int Neuropsychol Soc 1995;1:525-36.

11 Johnson DR, Sawyer AM, Meyers CA, et al. Early measures of cognitive function predict survival in patients with newly diagnosed glioblastoma. Neuro Oncol 2012;14:808-16.

12 Nys GMS, van Zandvoort MJE, de Kort PLM, et al. The prognostic value of domain-specific cognitive abilities in acute first-ever stroke. Neurology 2005;64:821-7.

13 Levine B, Schweizer TA, O'Connor C, et al. Rehabilitation of executive functioning in patients with frontal lobe brain damage with goal management training. Front Hum Neurosci 2011;5:9.

14 Zhang Y, Kensler TW, Cho CG, et al. Anticarcinogenic activities of sulforaphane and structurally related synthetic norbornyl isothiocyanates. Proc Natl Acad Sci U S A 1994;91:3147-50.

15 Singh K, Connors SL, Macklin EA, et al. Sulforaphane treatment of autism spectrum disorder (ASD). Proc Natl Acad Sci U S A 2014;111:15550-5.

16 Guerrero-Beltrán CE, Calderón-Oliver M, Pedraza-Chaverri J, et al. Protective effect of sulforaphane against oxidative stress: recent advances. Exp Toxicol Pathol 2012;64:503-8.

17 Chen $\mathrm{H}$, Wu J, Zhang J, et al. Protective effects of the antioxidant sulforaphane on behavioral changes and neurotoxicity in mice after the administration of methamphetamine. Psychopharmacology 2012;222:37-45

18 Wu J, Han J, Hou B, et al. Sulforaphane inhibits TGF- $\beta$-induced epithelial-mesenchymal transition of hepatocellular carcinoma cells via the reactive oxygen species-dependent pathway. Oncol Rep 2016;35:2977-83.

19 Chi X, Zhang R, Shen N, et al. Sulforaphane reduces apoptosis and oncosis along with protecting liver injury-induced ischemic reperfusion by activating the Nrf2/ARE pathway. Hepatol Int 2015;9:321-9.

20 Shiina A, Kanahara N, Sasaki T, et al. An open study of Sulforaphanerich broccoli sprout extract in patients with schizophrenia. Clin Psychopharmacol Neurosci 2015;13:62-7.

21 Dash PK, Zhao J, Orsi SA, et al. Sulforaphane improves cognitive function administered following traumatic brain injury. Neurosci Lett 2009;460:103-7.

22 Egan MF, Kojima M, Callicott JH, et al. The BDNF Val66Met polymorphism affects activity-dependent secretion of BDNF and human memory and hippocampal function. Cell 2003;112:257-69.

23 Jung RE, Yeo RA, Chiulli SJ, et al. Biochemical markers of cognition. Neuroreport 1999;10:3327-31. 
24 Yeo RA, Phillips JP, Jung RE, et al. Magnetic resonance spectroscopy detects brain injury and predicts cognitive functioning in children with brain injuries. J Neurotrauma 2006;23:1427-35.

25 Ariza $\mathrm{M}$, Junqué $\mathrm{C}$, Mataró $\mathrm{M}$, et al. Neuropsychological correlates of basal ganglia and medial temporal lobe NAA/Cho reductions in traumatic brain injury. Arch Neurol 2004;61:541-4.

26 Prediger RDS, Franco JL, Pandolfo P, et al. Differential susceptibility following beta-amyloid peptide-(1-40) administration in C57BL/6 and Swiss albino mice: Evidence for a dissociation between cognitive deficits and the glutathione system response. Behav Brain Res 2007;177:205-13.

27 Birzniece V, Bäckström T, Johansson I-M, et al. Neuroactive steroid effects on cognitive functions with a focus on the serotonin and GABA systems. Brain Res Rev 2006;51:212-39.

28 Zong X, Hu M, Li Z, et al. N-Acetylaspartate reduction in the medial prefrontal cortex following 8 weeks of risperidone treatment in firstepisode drug-naïve schizophrenia patients. Sci Rep 2015;5:9109.

29 Wu A, Ying Z, Gomez-Pinilla F, FJJon G-P. Dietary omega-3 fatty acids normalize BDNF levels, reduce oxidative damage, and counteract learning disability after traumatic brain injury in rats. $J$ Neurotrauma 2004;21:1457-67.

30 Sedlak TW, Nucifora LG, Koga M, et al. Sulforaphane augments glutathione and influences brain metabolites in human subjects: a clinical pilot study. Mol Neuropsychiatry 2018;3:214-22.

31 Huang C, Wu J, Chen D, et al. Effects of sulforaphane in the central nervous system. Eur J Pharmacol 2019;853:153-68.

32 Cryan JF, Dinan TG. Mind-altering microorganisms: the impact of the gut microbiota on brain and behaviour. Nat Rev Neurosci 2012;13:701-12.

33 Galland L. The gut microbiome and the brain. J Med Food 2014;17:1261-72.

34 Mayer EA, Tillisch K, Gupta A. Gut/Brain axis and the microbiota. J Clin Invest 2015;125:926-38.

35 Blander JM, Longman RS, Iliev ID, et al. Regulation of inflammation by microbiota interactions with the host. Nat Immunol 2017;18:851-60
36 Sharon G, Sampson TR, Geschwind DH, et al. The central nervous system and the gut microbiome. Cell 2016;167:915-32.

37 Yu Z, Morrison M. Improved extraction of PCR-quality community DNA from digesta and fecal samples. Biotechniques 2004;36:808-12.

38 Nasreddine ZS, Phillips NA, Bédirian V, et al. The Montreal cognitive assessment, MoCA: a brief screening tool for mild cognitive impairment. J Am Geriatr Soc 2005;53:695-9.

$39 \mathrm{Yu}$ J, Li J, Huang X. The Beijing version of the Montreal cognitive assessment as a brief screening tool for mild cognitive impairment: a community-based study. BMC Psychiatry 2012;12:156.

40 Socała K, Nieoczym D, Kowalczuk-Vasilev E, et al. Increased seizure susceptibility and other toxicity symptoms following acute sulforaphane treatment in mice. Toxicol Appl Pharmacol 2017;326:43-53.

41 Benedict RHB, Schretlen D, Groninger L, et al. Hopkins verbal learning test - revised: normative data and analysis of Inter-Form and test-retest reliability. Clin Neuropsychol 1998;12:43-55.

42 Bowie CR, Harvey PD. Administration and interpretation of the TRAIL making test. Nat Protoc 2006;1:2277-81.

43 White TSR. Neuropsychological assessment battery: psychometric and technical manual. Lutz, FL: Psychological Assessment Resources, 2003.

44 Lezak MD, Howieson DB, Loring DW, et al. Neuropsychological assessment. USA: Oxford University Press, 2004.

45 Anderson SW, Damasio H, Jones RD, et al. Wisconsin card sorting test performance as a measure of frontal lobe damage. J Clin Exp Neuropsychol 1991;13:909-22.

46 Benedict RHB, Schretlen D, Groninger L, et al. Revision of the brief visuospatial memory test: studies of normal performance, reliability, and validity. Psychol Assess 1996;8:145-53.

47 Miller E. Verbal fluency as a function of a measure of verbal intelligence and in relation to different types of cerebral pathology. $\mathrm{Br}$ J Clin Psychol 1984;23(Pt 1):53-7. 